\title{
Correction to: Structure of attractors for boundary maps associated to Fuchsian groups
}

\author{
Svetlana Katok ${ }^{1}$ • Ilie Ugarcovici ${ }^{2}$
}

Published online: 6 March 2018

(C) Springer Science+Business Media B.V., part of Springer Nature 2018

\section{Correction to: Geom Dedicata (2017) 191:171-198 https://doi.org/10.1007/s10711-017-0251-z}

The paper [2] studies the dynamics of a class of circle maps and their two-dimensional natural extensions built using the generators of a given cocompact and torsion-free Fuchsian group $\Gamma$. If $\mathbb{D}$ denotes the Poincaré unit disk model endowed with the standard hyperbolic metric, then $\Gamma \backslash \mathbb{D}$ is a compact surface of constant negative curvature and of a certain genus $g>1$. Most of the considerations and proofs in the paper were done for a special case of surface groups, those that admit a fundamental domain $\mathcal{F}$ given by a regular $(8 g-4)$-sided polygon. On p. 172 in the Introduction, we neglected to explain in the paragraph below equation (1.2) that, although not all surface groups admit such a fundamental domain, it is possible to reduce the general case to this special situation without affecting the results of the paper (see also [1, Appendix A]).

More precisely, given $\Gamma^{\prime} \backslash \mathbb{D}$ a compact surface of genus $g>1$, there exists a Fuchsian group $\Gamma$ such that:

(i) $\Gamma \backslash \mathbb{D}$ is a compact surface of the same genus $g$;

(ii) $\Gamma$ has a fundamental domain $\mathcal{F}$ given by a regular $(8 g-4)$-sided polygon;

(iii) By the Fenchel-Nielsen theorem [3] there exists an orientation preserving homeomorphism $h$ from $\overline{\mathbb{D}}$ onto $\overline{\mathbb{D}}$ such that $\Gamma^{\prime}=h \circ \Gamma \circ h^{-1}$.

The original article can be found online at https://doi.org/10.1007/s10711-017-0251-z.

$\bowtie \quad$ Ilie Ugarcovici

iugarcov@depaul.edu

Svetlana Katok

sxk37@psu.edu

1 Department of Mathematics, The Pennsylvania State University, University Park, PA 16802, USA

2 Department of Mathematical Sciences, DePaul University, Chicago, IL 60614, USA 
One can now extend the considerations described in the introductory section of the paper to any compact surface $\Gamma^{\prime} \backslash \mathbb{D}$, using the orientation preserving homeomorphism $h$ and the setting for $\Gamma \backslash \mathbb{D}$. Let

$$
T_{i}^{\prime}=h \circ T_{i} \circ h^{-1}, P_{i}^{\prime}=h\left(P_{i}\right) \text { and } Q_{i}^{\prime}=h\left(Q_{i}\right) .
$$

Then the set $\left\{T_{i}^{\prime}\right\}$ satisfies relations (1.3)-(1.5) and the order of the points $\left\{P_{i}^{\prime}\right\} \cup\left\{Q_{i}^{\prime}\right\}$ will be the same as for the set $\left\{P_{i}\right\} \cup\left\{Q_{i}\right\}$. The geodesics $P_{i}^{\prime} Q_{i+1}^{\prime}$ will produce the $(8 g-4)$-sided polygon $\mathcal{F}^{\prime}$ whose sides are identified by transformations $T_{i}^{\prime}$. Adler and Flatto [1, Appendix A] conclude that region $\mathcal{F}^{\prime}$ satisfies all the conditions of Poincaré's theorem, hence it is the fundamental domain for $\Gamma^{\prime}$.

The main object of study in our paper is the generalized Bowen-Series circle map $f_{A}$ : $S \rightarrow S$ given by (1.8)

$$
f_{\bar{A}}(x)=T_{i}(x) \text { if } A_{i} \leq x<A_{i+1},
$$

with the set of jump points $\bar{A}=\left\{A_{1}, A_{2}, \ldots, A_{8 g-4}\right\}$ satisfying the condition that $A_{i} \in$ $\left(P_{i}, Q_{i}\right), 1 \leq i \leq 8 g-4$. The corresponding two-dimensional extension map given by (1.9) is

$$
F_{\bar{A}}(x, y)=\left(T_{i}(x), T_{i}(y)\right) \text { if } A_{i} \leq y<A_{i+1} .
$$

Even though the main results of the paper (Theorems 1.2 and 1.3) were proved for the special situation of a genus $g$ compact surface $\Gamma \backslash \mathbb{D}$ that admits a regular $(8 g-4)$ sided fundamental region, the results remain true in full generality for an arbitrary genus $g$ compact surface $\Gamma^{\prime} \backslash \mathbb{D}$ with the set of $(8 g-4)$ generators $\left\{T_{i}^{\prime}\right\}$, the set of jump points $\bar{A}^{\prime}=\left\{A_{1}^{\prime}, A_{2}^{\prime}, \ldots, A_{8 g-4}^{\prime}\right\}$ with $A_{i}^{\prime}=h\left(A_{i}\right) \in\left(P_{i}^{\prime}, Q_{i}^{\prime}\right)$ and the corresponding maps:

$$
f_{\bar{A}^{\prime}}(x)=T_{i}^{\prime}(x) \quad \text { if } A_{i}^{\prime} \leq x<A_{i+1}^{\prime} ; \quad F_{\bar{A}^{\prime}}(x, y)=\left(T_{i}^{\prime}(x), T_{i}^{\prime}(y)\right) \quad \text { if } A_{i}^{\prime} \leq y<A_{i+1}^{\prime} .
$$

The orientation preserving homeomorphism $h: \overline{\mathbb{D}} \rightarrow \overline{\mathbb{D}}$ and the relations

$$
f_{\bar{A}^{\prime}}=h \circ f_{\bar{A}} \text { and } F_{\bar{A}^{\prime}}=(h \times h) \circ F_{\bar{A}}
$$

allow us to conclude that:

(a) A partition point $A_{i}^{\prime} \in\left(P_{i}^{\prime}, Q_{i}^{\prime}\right), 1 \leq i \leq 8 g-4$, satisfies the cycle property, i.e., there exist positive integers $m_{i}, k_{i}$ such that

$$
f_{\bar{A}^{\prime}}^{m_{i}}\left(T_{i}^{\prime} A_{i}^{\prime}\right)=f_{\bar{A}^{\prime}}^{k_{i}}\left(T_{i-1}^{\prime} A_{i}^{\prime}\right)
$$

if and only if the corresponding partition point $A_{i}=h^{-1}\left(A_{i}^{\prime}\right) \in\left(P_{i}, Q_{i}\right)$ satisfies the cycle property

$$
f_{\bar{A}}^{m_{i}}\left(T_{i} A_{i}\right)=f_{\bar{A}}^{k_{i}}\left(T_{i-1} A_{i}\right) .
$$

(b) A partition point $A_{i}^{\prime}$ satisfies the short cycle property

$$
f_{\bar{A}^{\prime}}\left(T_{i}^{\prime} A_{i}^{\prime}\right)=f_{\bar{A}^{\prime}}\left(T_{i-1}^{\prime} A_{i}^{\prime}\right)
$$

if and only if the corresponding partition point $A_{i}=h^{-1}\left(A_{i}^{\prime}\right)$ satisfies the short cycle property:

$$
f_{\bar{A}}\left(T_{i} A_{i}\right)=f_{\bar{A}}\left(T_{i-1} A_{i}\right)
$$


(c) If $\Omega_{\bar{A}}=\bigcap_{n=0}^{\infty} F_{\bar{A}}^{n}(\mathbb{S} \times \mathbb{S} \backslash \Delta)$ is the global attractor of the map $F_{\bar{A}}$, then $\Omega_{\bar{A}^{\prime}}=(h \times$ $h)\left(\Omega_{\bar{A}}\right)$ is the global attractor of the map $F_{\bar{A}^{\prime}}$. Also, if $\Omega_{\bar{A}}$ has finite rectangular structure, then $\Omega_{\bar{A}^{\prime}}$ has finite rectangular structure, since $h \times h$ preserves horizontal and vertical lines.

We would like to use this opportunity to also correct some misprints: on p. 173, last paragraph, the text "of the fundamental domain $\mathcal{F}$ " should read "of $\mathbb{D}$ "; on p. 193, in equation (7.2), the term " $A_{i}+1$ " should read " $A_{i+1}$ "; on p. 193, Proposition 7.1, the relations " $B_{i}=$ $T_{i} A_{i}$, and $C_{i}=T_{i-1} A_{i}$ " should read " $B_{i}=T_{\sigma(i-1)} A_{\sigma(i-1)}$, and $C_{i}=T_{\sigma(i+1)} A_{\sigma(i+1)+1}$."

\section{References}

1. Adler, R., Flatto, L.: Geodesic flows, interval maps, and symbolic dynamics. Bull. Am. Math. Soc. 25(2), 229-334 (1991)

2. Katok, S., Ugarcovici, I.: Structure of attractors for boundary maps associated to Fuchsian groups. Geom. Dedicata 191, 171-198 (2017). https://doi.org/10.1007/s10711-017-0251-z

3. Tukia, P.: On discrete groups of the unit disk and their isomorphisms. Ann. Acad. Sci. Fenn. Ser. A I. Math. 504, 5-44 (1972) 